Proceedings of the Edinburgh Mathematical Society (2004) 47, 155-162 (C)

DOI:10.1017/S001309150300052X Printed in the United Kingdom

\title{
A POLYNOMIAL MODEL FOR THE DOUBLE-LOOP SPACE OF AN EVEN SPHERE
}

\author{
YASUHIKO KAMIYAMA \\ Department of Mathematics, University of the Ryukyus, Nishihara-Cho, \\ Okinawa 903-0213, Japan (kamiyama@sci.u-ryukyu.ac.jp)
}

(Received 12 June 2003)

\begin{abstract}
It is well known that $\Omega^{2} S^{2 n+1}$ is approximated by $\operatorname{Rat}_{k}\left(\mathbb{C} P^{n}\right)$, the space of based holomorphic maps of degree $k$ from $S^{2}$ to $\mathbb{C} P^{n}$. In this paper we construct a space $G_{k}^{n}$ which is an analogue of $\operatorname{Rat}_{k}\left(\mathbb{C} P^{n}\right)$, and prove that under the natural map $j_{k}: G_{k}^{n} \rightarrow \Omega^{2} S^{2 n}, G_{k}^{n}$ approximates $\Omega^{2} S^{2 n}$.
\end{abstract}

Keywords: rational function; even sphere; loop space

2000 Mathematics subject classification: Primary 55P35

\section{Introduction}

Let $\operatorname{Rat}_{k}\left(\mathbb{C} P^{n}\right)$ denote the space of based holomorphic maps of degree $k$ from the Riemannian sphere $S^{2}=\mathbb{C} \cup \infty$ to the complex projective space $\mathbb{C} P^{n}$. The basepoint condition we assume is that $f(\infty)=[1, \ldots, 1]$. Such holomorphic maps are given by rational functions:

$$
\begin{aligned}
\operatorname{Rat}_{k}\left(\mathbb{C} P^{n}\right)=\left\{\left(p_{0}(z), \ldots, p_{n}(z)\right): \text { each } p_{i}(z) \text { is a monic polynomial over } \mathbb{C}\right. \\
\text { of degree } \left.k \text { and such that there are no roots common to all } p_{i}(z)\right\} .
\end{aligned}
$$

There is an inclusion

$$
i_{k}: \operatorname{Rat}_{k}\left(\mathbb{C} P^{n}\right) \hookrightarrow \Omega_{k}^{2} \mathbb{C} P^{n} \simeq \Omega^{2} S^{2 n+1} .
$$

Segal [9] proved that $i_{k}$ is a homotopy equivalence up to dimension $k(2 n-1)$. Later, the stable homotopy type of $\operatorname{Rat}_{k}\left(\mathbb{C} P^{n}\right)$ was described in $[\mathbf{6}]$ and $[\mathbf{7}]$ as follows. Let

$$
\Omega^{2} S^{2 n+1} \underset{s}{\simeq} \bigvee_{1 \leqslant q} D_{q}\left(S^{2 n-1}\right)
$$

be Snaith's stable splitting of $\Omega^{2} S^{2 n+1}$. Then

$$
\operatorname{Rat}_{k}\left(\mathbb{C} P^{n}\right) \underset{s}{\simeq} \bigvee_{q=1}^{k} D_{q}\left(S^{2 n-1}\right) .
$$


(We can rewrite this using the fact $[\mathbf{5}]$ that $D_{q}\left(S^{2 n-1}\right) \simeq \Sigma^{2 q(n-1)} D_{q}\left(S^{1}\right)$.) In particular,

$$
i_{k *}: H_{*}\left(\operatorname{Rat}_{k}\left(\mathbb{C} P^{n}\right) ; \boldsymbol{Z}\right) \rightarrow H_{*}\left(\Omega^{2} S^{2 n+1} ; \boldsymbol{Z}\right)
$$

is injective.

Results of [6], [7] and [9] imply that $\operatorname{Rat}_{k}\left(\mathbb{C} P^{n}\right)$ approximates $\Omega^{2} S^{2 n+1}$. On the other hand, considering the double-loop space of an even sphere, we naturally encounter the following problem: how to construct spaces $G_{k}^{n}$ which approximate $\Omega^{2} S^{2 n}$. Moreover, we study the stable homotopy type of $G_{k}^{n}$.

In special cases an answer is known. We set

$$
\operatorname{RRat}_{k}\left(\mathbb{C} P^{n}\right)=\left\{\left(p_{0}(z), \ldots, p_{n}(z)\right) \in \operatorname{Rat}_{k}\left(\mathbb{C} P^{n}\right): \text { each } p_{i}(z) \text { has real coefficients }\right\} .
$$

Let $\operatorname{Map}_{k}^{T}\left(\mathbb{C} P^{1}, \mathbb{C} P^{n}\right)$ denote the space of continuous basepoint-preserving conjugationequivariant maps of degree $k$ from $\mathbb{C} P^{1}$ to $\mathbb{C} P^{n}$. It is proved in [8] that

$$
\operatorname{Map}_{k}^{T}\left(\mathbb{C} P^{1}, \mathbb{C} P^{n}\right) \simeq \Omega S^{n} \times \Omega^{2} S^{2 n+1} \quad(n \geqslant 1) .
$$

Hence, there is an inclusion

$$
h_{k}: \operatorname{RRat}_{k}\left(\mathbb{C} P^{n}\right) \hookrightarrow \operatorname{Map}_{k}^{T}\left(\mathbb{C} P^{1}, \mathbb{C} P^{n}\right) \simeq \Omega S^{n} \times \Omega^{2} S^{2 n+1} .
$$

The map $h_{k}$ is a homotopy equivalence up to dimension $(k+1)(n-1)-1$. Moreover, $\operatorname{RRat}_{k}\left(\mathbb{C} P^{n}\right)$ is stably homotopy equivalent to the collection of stable summands in $\Omega S^{n} \times \Omega^{2} S^{2 n+1}$ of weight less than or equal to $k$. Here we define the weight of stable summands in $\Omega S^{n}$ as usual, but those in $\Omega^{2} S^{2 n+1}$ we define as being twice the usual one. Hence, in the situation where $\Omega^{2} S^{2 n} \simeq \Omega S^{2 n-1} \times \Omega^{2} S^{4 n-1}$ holds, we can say that $\operatorname{RRat}_{k}\left(\mathbb{C} P^{2 n-1}\right)$ is a model which approximates $\Omega^{2} S^{2 n}$. Such a situation holds either (i) when it is localized at an odd prime, or (ii) when $n=1,2$ or 4 .

In this paper we construct spaces $G_{k}^{n}$ which approximate $\Omega^{2} S^{2 n}$ for all $n$ without localization.

Definition 1.1. For $n \geqslant 1$, let $G_{k}^{n}$ denote the space consisting of all $(n+1)$-tuples $\left(p_{0}(z), \ldots, p_{n}(z)\right)$ of monic polynomials over $\mathbb{C}$ of degree $k$ and such that if $p_{0}(\alpha)=\cdots=$ $p_{n-1}(\alpha)=0$ for some $\alpha \in \mathbb{C}$, then $p_{n}(\alpha) \notin \mathbb{R}$.

We have $G_{1}^{n} \simeq S^{2 n-2}$ (cf. Lemma $2.3(\mathrm{i})$ ). Define a map

$$
j_{k}: G_{k}^{n} \rightarrow \Omega^{2} S^{2 n}
$$

as follows. We embed $\mathbb{R} \hookrightarrow \mathbb{C}^{n+1}$ by

$$
r \mapsto(\underbrace{0, \ldots, 0}_{n \text { times }}, r) .
$$

Note that $\mathbb{R}^{+}$, the group of positive real numbers, acts on $\mathbb{C}^{n+1}-\mathbb{R}$ so that

$$
\left(\mathbb{C}^{n+1}-\mathbb{R}\right) / \mathbb{R}^{+} \simeq \mathbb{C}^{n+1}-\mathbb{R} \simeq S^{2 n} .
$$


Then $j_{k}$ is defined to be the composite of maps

$$
G_{k}^{n} \hookrightarrow \Omega^{2}\left(\left(\mathbb{C}^{n+1}-\mathbb{R}\right) / \mathbb{R}^{+}\right) \simeq \Omega^{2}\left(\mathbb{C}^{n+1}-\mathbb{R}\right) \simeq \Omega^{2} S^{2 n} .
$$

For $n \geqslant 2$, let

$$
\Omega^{2} S^{2 n} \underset{s}{\simeq} \bigvee_{1 \leqslant q} D_{q}\left(S^{2 n-2}\right)
$$

be Snaith's stable splitting of $\Omega^{2} S^{2 n}$.

From results of $[\mathbf{3}]$ and $[\mathbf{5}]$, there is a stable homotopy equivalence

$$
D_{q}\left(S^{2 n-2}\right) \underset{s}{\simeq} S^{2 q(n-1)} \vee \bigvee_{i=1}^{[q / 2]} \Sigma^{2 q(n-1)} D_{i}\left(S^{1}\right) .
$$

Our main results are the following two theorems.

Theorem A. For $n \geqslant 2$, there is a stable homotopy equivalence

$$
G_{k}^{n} \underset{s}{\simeq} \bigvee_{q=1}^{k} D_{q}\left(S^{2 n-2}\right)
$$

\section{Theorem B.}

(i) For $n \geqslant 2$, the map $j_{k}: G_{k}^{n} \rightarrow \Omega^{2} S^{2 n}$ induces isomorphisms in homology groups in dimensions less than or equal to $(k+1)(2 n-2)-1$. Hence, $j_{k}$ induces isomorphisms in homotopy groups in dimensions less than or equal to $(k+1)(2 n-2)-2$.

(ii) For $n \geqslant 2, j_{k *}: H_{*}\left(G_{k}^{n} ; \boldsymbol{Z}\right) \rightarrow H_{*}\left(\Omega^{2} S^{2 n} ; \boldsymbol{Z}\right)$ is injective.

Finally, we study the case $n=1$. Recall that Brockett and Segal $[\mathbf{2}, \mathbf{9}]$ showed that $\operatorname{RRat}_{k}\left(\mathbb{C} P^{1}\right)$ has $k+1$ connected components such that

$$
\operatorname{RRat}_{k}\left(\mathbb{C} P^{1}\right) \simeq \coprod_{q=0}^{k} \operatorname{Rat}_{m i n}(q, k-q)\left(\mathbb{C} P^{1}\right) .
$$

Theorem C. There is a homotopy equivalence

$$
G_{k}^{1} \simeq \operatorname{RRat}_{k}\left(\mathbb{C} P^{1}\right) .
$$

\section{Proofs of Theorems A, B and C}

In order to prove Theorem A, we first prove the following proposition.

Proposition 2.1. Let $p$ be a prime. Then, as a vector space, $H_{*}\left(G_{k}^{n} ; \boldsymbol{Z} / p\right)$ is isomorphic to the subspace of $H_{*}\left(\Omega^{2} S^{2 n} ; \boldsymbol{Z} / p\right)$ spanned by monomials of weight less than or equal to $k$. Here we define the weight of the (torsion-free) generators of $H_{2 n-2}\left(\Omega^{2} S^{2 n} ; \boldsymbol{Z}\right)$ and $H_{4 n-3}\left(\Omega^{2} S^{2 n} ; \boldsymbol{Z}\right)$ to be 1 and 2 , respectively (cf. (1.1)). 
The proposition is proved as follows. First, by constructing homology classes explicitly, we find a lower bound for the $\bmod p$ homology of $G_{k}^{n}$ (cf. Proposition 2.2). Next, considering a geometrical resolution of a resultant, we construct a spectral sequence of Vassiliev type. The spectral sequence converges to the $\bmod p$ homology of $G_{k}^{n}$ and the $E^{1}$ term coincides with the lower bound. Hence, the spectral sequence collapses at the $E^{1}$ term and the lower bound is actually an upper bound (cf. Proposition 2.4).

Proposition 2.2. Every element of $H_{*}\left(\Omega^{2} S^{2 n} ; \boldsymbol{Z} / p\right)$ of weight less than or equal to $k$ is in the image of $j_{k *}$. Hence, these elements are a lower bound for $H_{*}\left(G_{k}^{n} ; \boldsymbol{Z} / p\right)$.

In order to prove Proposition 2.2, we first prove the following lemma.

\section{Lemma 2.3.}

(i) The (torsion-free) generator of $H_{2 n-2}\left(\Omega^{2} S^{2 n} ; \boldsymbol{Z}\right)$ is in the image of $j_{1 *}$.

(ii) The (torsion-free) generator of $H_{4 n-3}\left(\Omega^{2} S^{2 n} ; \boldsymbol{Z}\right)$ is in the image of $j_{2 *}$.

\section{Proof.}

(i) We embed $\mathbb{R} \hookrightarrow \mathbb{C}^{n}$ by

$$
r \mapsto(\underbrace{0, \ldots, 0}_{n-1 \text { times }}, r)
$$

Define a homeomorphism

$$
f: G_{1}^{n} \stackrel{\cong}{\longrightarrow} \times\left(\mathbb{C}^{n}-\mathbb{R}\right)
$$

by

$$
f\left(z+\alpha_{0}, \ldots, z+\alpha_{n}\right)=\left(\alpha_{0},\left(\alpha_{1}-\alpha_{0}, \ldots, \alpha_{n}-\alpha_{0}\right)\right) .
$$

Then $G_{1}^{n} \simeq S^{2 n-2}$. Let $u_{2 n-2}$ be the generator of $H_{2 n-2}\left(G_{1}^{n} ; \boldsymbol{Z}\right)$. Then it is easy to see that $j_{1 *}\left(u_{2 n-2}\right)$ generates $H_{2 n-2}\left(\Omega^{2} S^{2 n} ; \boldsymbol{Z}\right)$.

(ii) Let $B^{n}$ be the space consisting of all $n$-tuples $\left(p_{0}(z), \ldots, p_{n-1}(z)\right)$ of monic polynomials over $\mathbb{C}$ of degree 2 and such that

$$
\left(p_{0}(z), \ldots, p_{n-1}(z)\right) \neq\left((z+\alpha)^{2}, \ldots,(z+\alpha)^{2}\right) \text { for any } \alpha \in \mathbb{C} .
$$

There is an embedding $s: B^{n} \rightarrow G_{2}^{n}$ defined by

$$
s\left(p_{0}(z), \ldots, p_{n-1}(z)\right)=\left(p_{0}(z), \ldots, p_{n}(z)\right),
$$

where $p_{n}(z)$ is chosen according to $p_{i}(z)(0 \leqslant i \leqslant n-1)$ as follows: we choose the imaginary part of the constant term of $p_{n}(z)$ near $+\infty$ so that $p_{n}(\alpha) \notin \mathbb{R}$ for any $\alpha$, a root of $p_{i}(z)$ for some $0 \leqslant i \leqslant n-1$.

Since $B^{n} \cong \mathbb{C}^{2 n}-\mathbb{C} \simeq S^{4 n-3}$, there is an element $v_{4 n-3} \in H_{4 n-3}\left(G_{2}^{n} ; \boldsymbol{Z}\right)$. It is easy to see that $j_{2 *}\left(v_{4 n-3}\right)$ generates $H_{4 n-3}\left(\Omega^{2} S^{2 n} ; \boldsymbol{Z}\right)$. This completes the proof of Lemma 2.3 . 
Proof of Proposition 2.2. By an argument quite similar to that found in [1], we have a loop sum

$$
*: H_{i}\left(G_{k}^{n} ; \boldsymbol{Z} / p\right) \otimes H_{j}\left(G_{k^{\prime}}^{n} ; \boldsymbol{Z} / p\right) \rightarrow H_{i+j}\left(G_{k+k^{\prime}}^{n} ; \boldsymbol{Z} / p\right)
$$

and the first Dyer-Lashof operation

$$
Q_{1}: H_{i}\left(G_{k}^{n} ; \boldsymbol{Z} / p\right) \rightarrow H_{i p+p-1}\left(G_{k p}^{n} ; \boldsymbol{Z} / p\right)
$$

that are compatible with those in $H_{*}\left(\Omega^{2} S^{2 n} ; \boldsymbol{Z} / p\right)$.

The structure of $H_{*}\left(\Omega^{2} S^{2 n} ; \boldsymbol{Z} / p\right)$ is given as follows (cf. [4]).

(i) For $p=2$,

$$
H_{*}\left(\Omega^{2} S^{2 n} ; \boldsymbol{Z} / 2\right) \cong \boldsymbol{Z} / 2\left[u_{2 n-2}, Q_{1}\left(u_{2 n-2}\right), \ldots, Q_{1} \cdots Q_{1}\left(u_{2 n-2}\right), \ldots\right] .
$$

(ii) For an odd prime $p$,

$$
H_{*}\left(\Omega^{2} S^{2 n} ; \boldsymbol{Z} / p\right) \cong H_{*}\left(\Omega S^{2 n-1} ; \boldsymbol{Z} / p\right) \otimes H_{*}\left(\Omega^{2} S^{4 n-1} ; \boldsymbol{Z} / p\right) .
$$

Moreover, $H_{*}\left(\Omega S^{2 n-1} ; \boldsymbol{Z} / p\right) \cong \boldsymbol{Z} / p\left[u_{2 n-2}\right]$ and

$$
\begin{aligned}
& H_{*}\left(\Omega^{2} S^{4 n-1} ; \boldsymbol{Z} / p\right) \cong \bigwedge\left(v_{4 n-3}, Q_{1}\left(v_{4 n-3}\right), \ldots, Q_{1} \cdots Q_{1}\left(v_{4 n-3}\right), \ldots\right) \\
& \otimes \boldsymbol{Z} / p\left[\beta Q_{1}\left(v_{4 n-3}\right), \ldots, \beta Q_{1} \cdots Q_{1}\left(v_{4 n-3}\right), \ldots\right]
\end{aligned}
$$

where $\beta$ is the $\bmod p$ Bockstein operation.

By Lemma 2.3, $u_{2 n-2}$ is in the image of $j_{1 *}$ and $v_{4 n-3}$ is in the image of $j_{2 *}$. Hence, from the structure of $H_{*}\left(\Omega^{2} S^{2 n} ; \boldsymbol{Z} / p\right)$, every element of $H_{*}\left(\Omega^{2} S^{2 n} ; \boldsymbol{Z} / p\right)$ of weight less than or equal to $k$ is constructed in $H_{*}\left(G_{k}^{n} ; \boldsymbol{Z} / p\right)$. This completes the proof of Proposition 2.2.

Proposition 2.4. The lower bound of Proposition 2.2 is actually an upper bound.

Proof. We prove the proposition along the lines of [10, p. 151]. For a locally compact space $X$, let $\bar{X}$ denote the one-point compactification of $X, \bar{X}=X \cup\{\infty\}$, and let $\bar{H}_{*}(X ; \boldsymbol{Z})$ be the Borel-Moore homology group $\bar{H}_{*}(X ; \boldsymbol{Z})=\tilde{H}_{*}(\bar{X} ; \boldsymbol{Z})$.

We regard $\mathbb{C}^{k(n+1)}$ as the space consisting of all $(n+1)$-tuples $\left(p_{0}(z), \ldots, p_{n}(z)\right)$ of monic polynomials over $\mathbb{C}$ of degree $k$. Let $\Sigma_{k}^{n}$ be the complement of $G_{k}^{n}$ in $\mathbb{C}^{k(n+1)}$. Thus

$$
\begin{aligned}
\Sigma_{k}^{n}=\left\{\left(p_{0}(z), \ldots, p_{n}(z)\right) \in \mathbb{C}^{k(n+1)}: p_{0}(\alpha)=\cdots=\right. & p_{n-1}(\alpha)=0 \\
& \text { and } \left.p_{n}(\alpha) \in \mathbb{R} \text { for some } \alpha \in \mathbb{C}\right\} .
\end{aligned}
$$

From the Alexander duality, there is a natural isomorphism

$$
\tilde{H}^{*}\left(G_{k}^{n} ; \boldsymbol{Z}\right) \cong \bar{H}_{2 k(n+1)-1-*}\left(\Sigma_{k}^{n} ; \boldsymbol{Z}\right)
$$

and so we study $\bar{H}_{*}\left(\sum_{k}^{n} ; \boldsymbol{Z}\right)$. 
Let $I: \mathbb{C} \rightarrow \mathbb{C}^{k}$ be the Veronese embedding $I(z)=\left(z, z^{2}, \ldots, z^{k}\right)$. Let $f=$ $\left(p_{0}(z), \ldots, p_{n}(z)\right) \in \Sigma_{k}^{n}$ and suppose that $p_{0}(z), \ldots, p_{n-1}(z)$ have at least $d$ distinct common roots $\left\{\alpha_{1}, \ldots, \alpha_{d}\right\} \subset \mathbb{C}$ which satisfy $p_{n}\left(\alpha_{i}\right) \in \mathbb{R}(1 \leqslant i \leqslant d)$. We denote by $\Delta\left(f,\left\{\alpha_{1}, \ldots, \alpha_{d}\right\}\right) \subset \mathbb{C}^{k}$ the open simplex in $\mathbb{C}^{k}$ with vertices $\left\{I\left(\alpha_{1}\right), \ldots, I\left(\alpha_{d}\right)\right\}$. (Note that since $d \leqslant k$, the points $I\left(\alpha_{1}\right), \ldots, I\left(\alpha_{d}\right)$ are in general position.) Define a geometrical resolution $\tilde{\Sigma}_{k}^{n}$ of $\Sigma_{k}^{n}$ by

$$
\tilde{\Sigma}_{k}^{n}=\bigcup_{f \in \Sigma_{k}^{n} ;\left\{\alpha_{1}, \ldots, \alpha_{d}\right\}}\{f\} \times \Delta\left(f,\left\{\alpha_{1}, \ldots, \alpha_{d}\right\}\right) \subset \Sigma_{k}^{n} \times \mathbb{C}^{k} .
$$

The first projection defines an open proper map $\pi: \tilde{\Sigma}_{k}^{n} \rightarrow \Sigma_{k}^{n}$, and this induces a map between the one-point compactification spaces

$$
\bar{\pi}: \overline{\tilde{\Sigma}}_{k}^{n} \rightarrow \bar{\Sigma}_{k}^{n} .
$$

It is known [10] that the map $\bar{\pi}$ is a homotopy equivalence. Define subspaces $F_{s} \subset \bar{\Sigma}_{k}^{n}$ by

$$
F_{s}= \begin{cases}\{\infty\} \cup \underset{f \in \Sigma_{k}^{n} ;\left\{\alpha_{1}, \ldots, \alpha_{d}\right\}, d \leqslant s}{\bigcup}\{f\} \times \Delta\left(f,\left\{\alpha_{1}, \ldots, \alpha_{d}\right\}\right) & \text { if } s \geqslant 1, \\ \{\infty\} & \text { if } s=0 .\end{cases}
$$

There is an increasing filtration

$$
F_{0}=\{\infty\} \subset F_{1} \subset F_{2} \subset \cdots \subset F_{k}=\bar{\Sigma}_{k}^{n} \simeq \bar{\Sigma}_{k}^{n}
$$

and this induces a spectral sequence

$$
E_{s, t}^{1}=\bar{H}_{s+t}\left(F_{s}-F_{s-1} ; \boldsymbol{Z}\right) \Rightarrow \bar{H}_{s+t}\left(\tilde{\Sigma}_{k}^{n} ; \boldsymbol{Z}\right) \cong \bar{H}_{s+t}\left(\Sigma_{k}^{n} ; \boldsymbol{Z}\right) .
$$

$F_{s}-F_{s-1}$ is the space of a fibre bundle which is a fibred product of the following two bundles. The two bundles have common base $C_{s}(\mathbb{C})$, where $C_{s}(\mathbb{C})$ denotes the configuration space of unordered $s$-tuples of distinct points in $\mathbb{C}$.

(i) The first bundle has an open $(s-1)$-dimensional simplex as a fibre.

(ii) The second bundle is an affine $\left(\left(\mathbb{C}^{k-s}\right)^{n+1} \times \mathbb{R}^{s}\right)$ bundle. The fibre over a collection $\left\{\alpha_{1}, \ldots, \alpha_{s}\right\} \in C_{s}(\mathbb{C})$ consists of $\left(\left(p_{0}(z), \ldots, p_{n}(z)\right),\left(r_{1}, \ldots, r_{s}\right)\right)$, where $\operatorname{deg} p_{i}(z)=$ $k(0 \leqslant i \leqslant n), p_{i}(z)(0 \leqslant i \leqslant n-1)$ has roots $\alpha_{1}, \ldots, \alpha_{s}$ and $p_{n}\left(\alpha_{j}\right)=r_{j}$ $(1 \leqslant j \leqslant s)$.

Consider a real $s$-dimensional vector bundle over $C_{s}(\mathbb{C})$ with fibre over a collection $\left\{\alpha_{1}, \ldots, \alpha_{s}\right\} \in C_{s}(\mathbb{C})$ being the space of functions on its points. The local system of the vector bundle is locally isomorphic to $\boldsymbol{Z}$ but changes the orientation over the loops defining odd permutations. Note that the bundles (i) and (ii) have this local system. Hence, by the Thom and Poincaré isomorphisms,

$$
E_{s, t}^{1}= \begin{cases}H^{2(k-s)(n+1)+3 s-t-1}\left(C_{s}(\mathbb{C}) ; \boldsymbol{Z}\right) & 1 \leqslant s \leqslant k, \\ 0 & \text { otherwise }\end{cases}
$$


Let $1 \leqslant *$. From the Alexander duality, we have

$$
\begin{aligned}
\operatorname{dim} H_{*}\left(G_{k}^{n} ; \boldsymbol{Z} / p\right) & \leqslant \sum_{s=1}^{k} \operatorname{dim} H_{*-2 s(n-1)}\left(C_{s}(\mathbb{C}) ; \boldsymbol{Z} / p\right) \\
& =\sum_{s=1}^{k} \operatorname{dim} H_{*}\left(\Sigma^{2 s(n-1)}\left(C_{s}(\mathbb{C}) \vee S^{0}\right) ; \boldsymbol{Z} / p\right)
\end{aligned}
$$

Since $D_{s}\left(S^{2 n-2}\right) \simeq \Sigma^{2 s(n-1)}\left(C_{s}(\mathbb{C}) \vee S^{0}\right)($ cf. [5] $)$, we have

$$
\operatorname{dim} H_{*}\left(G_{k}^{n} ; \boldsymbol{Z} / p\right) \leqslant \sum_{s=1}^{k} \operatorname{dim} H_{*}\left(D_{s}\left(S^{2 n-2}\right) ; \boldsymbol{Z} / p\right) .
$$

This completes the proof of Proposition 2.4, and, consequently, of Proposition 2.1.

Proof of Theorem A. Let $f_{k}$ be the stable map given by the composite of maps

$$
f_{k}: G_{k}^{n} \stackrel{j_{k}}{\longrightarrow} \Omega^{2} S^{2 n} \underset{s}{\simeq} \bigvee_{1 \leqslant q} D_{q}\left(S^{2 n-2}\right) \rightarrow \bigvee_{q=1}^{k} D_{q}\left(S^{2 n-2}\right)
$$

Note that $f_{k}$ is compatible with the homology splitting by weights. Then, using Proposition 2.1, we see that $f_{k}$ induces an isomorphism in homology, hence is a stable homotopy equivalence. This completes the proof of Theorem A.

\section{Proof of Theorem B.}

(i) Among elements of $H_{*}\left(\Omega^{2} S^{2 n} ; \boldsymbol{Z} / p\right)$ which are not contained in $\operatorname{Im} j_{k *}$, the element of least degree is $u_{2 n-2}^{k+1}$ (cf. Theorem A). Hence, the homological assertion holds. Since $G_{k}^{n}$ and $\Omega^{2} S^{2 n}$ are simply connected for $n \geqslant 2$, the homotopical assertion follows from the Whitehead Theorem.

(ii) Part (ii) is clear from Theorem A. This completes the proof of Theorem B.

Proof of Theorem C. Let $\left(p_{0}(z), p_{1}(z)\right) \in G_{k}^{1}$. If $p_{0}(\alpha)=0$, then we have $p_{1}(\alpha) \in$ $H_{+}$or $H_{-}$, where $H_{+}$(respectively, $H_{-}$) is the open upper (respectively, lower) halfplane. If $p_{1}(\alpha) \in H_{+}$(respectively, $H_{-}$), then we give the sign '+' (respectively, '-') to $\alpha$. Let $X_{k}$ be the space of unordered collections $\left\{\alpha_{1}, \ldots, \alpha_{k}\right\}$ of $k$ points in $\mathbb{C}$ such that each $\alpha_{i}$ has sign '+' or '-' with the following condition: if $\alpha_{i}$ and $\alpha_{j}$ have the same sign, then we allow $\alpha_{i}=\alpha_{j}$, but if they have opposite sign, then we do not allow $\alpha_{i}=\alpha_{j}$. It is clear that $G_{k}^{1} \simeq X_{k}$. Let $\left\{\beta_{1}, \ldots, \beta_{q}, \gamma_{1}, \ldots, \gamma_{k-q}\right\} \in X_{k}$, where $\beta_{i}$ has sign '+' and $\gamma_{i}$ has sign ' - '. We consider a pair of polynomials $\left(q_{0}(z), q_{1}(z)\right)$ defined by

$$
q_{0}(z)=\prod_{i=1}^{q}\left(z-\beta_{i}\right) \quad \text { and } \quad q_{1}(z)=\prod_{i=1}^{k-q}\left(z-\gamma_{i}\right) .
$$


Using the division algorithm we change $\left(q_{0}(z), q_{1}(z)\right)$ to an element of

$$
\operatorname{Rat}_{\min (q, k-q)}\left(\mathbb{C} P^{1}\right) .
$$

Then we see that $X_{k}$ has $k+1$ connected components so that

$$
X_{k} \simeq \coprod_{q=0}^{k} \operatorname{Rat}_{\min (q, k-q)}\left(\mathbb{C} P^{1}\right) .
$$

Now Theorem C follows from (1.2). This completes the proof of Theorem C.

Acknowledgements. Y.K. is indebted to Fred Cohen for numerous helpful conversations concerning configuration spaces.

\section{References}

1. C. P. Boyer and B. M. Mann, Monopoles, non-linear $\sigma$ models, and two-fold loop spaces, Commun. Math. Phys. 115 (1988), 571-594.

2. R. I. BRockett, Some geometric questions in the theory of linear systems, IEEE Trans. Automatic Control 21 (1976), 449-455.

3. E. H. Brown and F. P. Peterson, On the stable decomposition of $\Omega^{2} S^{r+2}$, Trans. Am. Math. Soc. 243 (1978), 287-298.

4. F. R. Cohen, T. J. LADA AND J. P. MaY, The homology of iterated loop spaces, Lecture Notes in Mathematics, vol. 533 (Springer, 1976).

5. F. R. Cohen, M. E. Mahowald and R. J. Milgram, The stable decomposition for the double loop space of a sphere, in Algebraic and geometric topology (ed. R. J. Milgram), Proc. Symp. Pure Mathematics, vol. 32, part 2, pp. 225-228 (American Mathematical Society, Providence, RI, 1978).

6. F. R. Cohen, R. L., Cohen, B. M. Mann and R. J. Milgram, The topology of rational functions and divisors of surfaces, Acta Math. 166 (1991), 163-221.

7. F. R. Cohen, R. L. Cohen, B. M. Mann and R. J. Milgram, The homotopy type of rational functions, Math. Z. 213 (1993), 37-47.

8. Y. Kamiyama, Spaces of real rational functions with common roots, preprint (2002).

9. G. B. Segal, The topology of spaces of rational functions, Acta Math. 143 (1979), 39-72.

10. V. A. VASsiliev, Complements of discriminants of smooth maps: topology and applications, Translations of Mathematical Monographs, vol. 98 (American Mathematical Society, Providence, RI, 1994). 